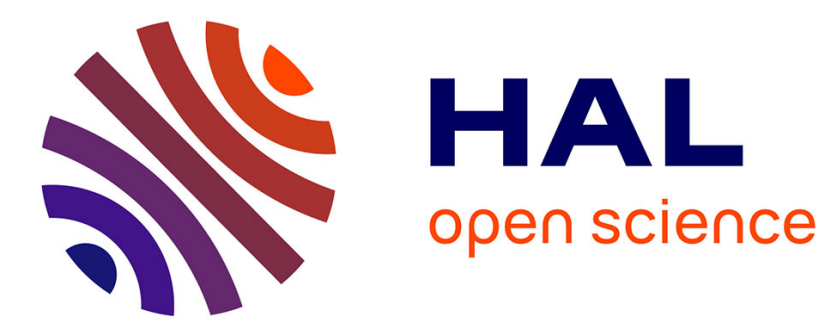

\title{
Le recours aux experts, un mode de rationalisation des pratiques judiciaires?
}

Laurence Dumoulin

\section{To cite this version:}

Laurence Dumoulin. Le recours aux experts, un mode de rationalisation des pratiques judiciaires?. Politiques et Management public, 2005, 23 (3), pp.145-159. halshs-00151023

\section{HAL Id: halshs-00151023 \\ https://shs.hal.science/halshs-00151023}

Submitted on 1 Jun 2007

HAL is a multi-disciplinary open access archive for the deposit and dissemination of scientific research documents, whether they are published or not. The documents may come from teaching and research institutions in France or abroad, or from public or private research centers.
L'archive ouverte pluridisciplinaire HAL, est destinée au dépôt et à la diffusion de documents scientifiques de niveau recherche, publiés ou non, émanant des établissements d'enseignement et de recherche français ou étrangers, des laboratoires publics ou privés. 


\title{
«Le recours aux experts, un mode de rationalisation des pratiques judiciaires ? »
}

\author{
Par \\ Laurence Dumoulin \\ Chargée de recherches ISP / CNRS, ENS Cachan \\ Publié dans $P M P, 2005$, vol.23, n³, pp.145-159
}

\section{RESUME :}

Cet article part du constat du développement de la rhétorique et des pratiques managériales dans l'espace judiciaire. Or, l'émergence de la question de "l'efficacité" appliquée à ce secteur est relativement récente. Ce n'est que dans les années 1980 et plus encore 1990, sous l'impact d'un mouvement global de modernisation des services publics notamment, qu'une forme de managérialisation de la justice commence à apparaître. Dès lors, cet article s'intéresse aux tensions spécifiques que génère la montée en puissance de cette logique managériale dans le milieu judiciaire, en particulier aux tensions qui naissent de la confrontation entre une logique gestionnaire et une culture centrée sur la juridicité. Il propose dans un premier temps de revenir sur ce mouvement et d'en analyser les modes de diffusion puis de centrer ensuite l'analyse sur un objet circonscrit, l' expertise judiciaire, pour mettre en évidence les ambiguïtés qui naissent de l'implantation d'une logique de "l'efficacité" dans un milieu encore très normé et très organisé autour de la mise en œuvre de procédures, procédures certes juridiques mais exprimant toujours une conception politique de ce que doit être la justice. L'expertise et les contradictions inhérentes à son développement percutent de plein fouet cette logique managériale et produisent des effets multiples : vecteur d'allongement des délais, d'augmentation des coûts et de multiplication des procédures, l'expertise est aussi, paradoxalement, un outil de régulation de l'organisation judiciaire dans un contexte de pénurie de moyens et de massification des contentieux. 
Dans sa présentation du budget de la justice pour l'année 2005, le Garde des Sceaux résumait ainsi les « quatre orientations principales de la loi d'orientation et de programmation pour la justice $»^{1}$ : « améliorer l'efficacité de la justice au service des citoyens et rapprocher la justice des justiciables; se donner les moyens de mieux exécuter les décisions pénales; traiter plus efficacement la délinquance des mineurs; donner de nouveaux droits aux victimes et leur permettre de mieux défendre leurs intérêts $»^{2}$. Cette citation montre bien la demande qui est aujourd'hui formulée à l'adresse de la justice : être "efficace", voilà l'injonction supérieure, qui conditionne toute appréhension de la qualité de la justice mais aussi toute attribution de ressources budgétaires supplémentaires.

L'émergence de cette question de "l'efficacité" appliquée au secteur judiciaire est relativement récente, en tout cas sous cette forme. Certes, la question des lenteurs de la justice, pour ne citer que celle-ci, est récurrente, tout au long des XIXe et XXe siècles, et témoigne d'interrogations sur les modes de fonctionnement judiciaire. Mais l'éloge de la lenteur, de l'art judiciaire, d'une temporalité nécessairement distincte du temps social, venait alors répondre aux indignations et caricatures critiquant une justice lente, paresseuse voire dilatoire

Ce n'est que dans les années 1980 et plus encore 1990, sous l'impact d'un mouvement global de modernisation des services publics notamment, que la question de "l'efficacité" de la justice commence d'être posée, en termes de réponses à apporter aux questions ou préoccupations de l'usager. La "rhétorique de l'usager » est le levier, le support à partir duquel sera déployée une politique de modernisation ${ }^{3}$. La justice, en dépit des spécificités voire de l'exceptionnalité qu'elle revendique, est touchée par cette lame de fond, ce mouvement managérial qui touche les autres secteurs de l'action publique, et qui vient substituer à une légitimité de type juridique et centrée sur l'indépendance juridictionnelle une légitimité centrée sur "l'efficacité", c'est-à-dire sur la capacité à atteindre des objectifs prédéfinis. De la même façon que le droit lui-même est touché par un impératif d'efficacité ${ }^{4}$, la justice doit rendre des comptes. Ce n'est plus le passage de la justice - comme institution chargée d'appliquer et de faire respecter le droit - qui est en soi suffisant pour conférer à la justice sa légitimité. Encore faut-il que l'action de justice soit perçue comme "efficace" c'està-dire rationnelle sur le plan de l'utilisation des moyens alloués et qu'elle règle effectivement les problèmes sociaux et politiques, dans un délai qui permette la visibilité et l'efficacité de la sanction - tant à l'égard du coupable, de la victime que de l'opinion publique.

Dans cette contribution, nous voudrions utiliser le cas de l'expertise pour mettre en lumière les ambiguités qui travaillent le secteur judiciaire dès lors que la question de "l'efficacité" et de l'économie de moyens s'y trouve posée. En effet, les investigations et

\footnotetext{
${ }^{1}$ Cette loi dite LOPJ fixe le cadre d'action pour 2003-2007 en matière de justice. Il s'agit de la loi no 2002-1138 du 9 septembre 2002.

${ }^{2}$ Présentation générale du budget de la justice 2005, «Le budget de la justice 2005 : une action dans la durée », ministère de la Justice, p.4, consultable à l'adresse suivante : http://www.justice.gouv.fr/publicat/budget2005.pdf, 9/02/2005. C'est nous qui soulignons.

${ }^{3}$ Voir DUMOULIN L. et DELPEUCH T. (1997), «La Justice : émergence d'une rhétorique de l'usager» in WARIN P. (dir.), Quelle modernisation des services publics? Les usagers au cœur des réformes, Paris, La Découverte, p.103-129.

${ }^{4}$ Instrument de l'action politique (ce qu'il a toujours été), il devient aussi et de plus en plus instrument de l'action publique, dispositif d'organisation des programmes d'action publique, outil de gestion et d'opérationnalisation. Voir CHEVALLIER J. et LOSCHAK D. (1982), «Rationalité juridique et rationalité managériale dans l'administration française », Revue française d'administration publique, 24.
} 
recherches empiriques menées dans le cadre de notre thèse de doctorat ${ }^{5}$ font apparaître l'expertise comme un des instruments et facteurs d'adaptation de l'institution à une exigence accrue de rationalisation des modes d'organisation, et ce bien qu'elle soit consommatrice de davantage de ressources et synonyme de plus de complexité dans le procès. De surcroit, on observe une forte tension entre une conception politique (exprimée par le droit) de l'expertise, et les manifestations diverses d'une logique centrée sur la gestion et l'économie de moyens. Les difficiles ajustements de ces différentes logiques nous semblent emblématiques des tiraillements qui travaillent actuellement l'espace judiciaire dans cette entreprise de désingularisation des modes de faire judiciaires par le recours au management.

${ }^{5}$ DUMOULIN L. (2001), L'expertise comme nouvelle raison politique? Discours, usages et effets de l'expertise judiciaire, Thèse de doctorat en science politique, IEP / UPMF, Grenoble. 


\section{I / De la modernisation à la managérialisation}

L'émergence d'une logique de "l'efficacité" s'est manifestée depuis les années 1980 sous des formes diverses qui, bien entendu sont liées mais que nous distinguerons ici à des fins analytiques. Prenant d'abord les traits d'une politique de modernisation de l'administration judiciaire passant par une amélioration de l'organisation et du fonctionnement des juridictions, la recherche de "l'efficacité" a ensuite procédé de l'invention de nouvelles formes judiciaires ou du réinvestissement de procédures négligées jusque-là et touchant aux modes d'exercice de la justice. Ces nombreuses réformes de justice destinées à résorber le malaise voire la crise qui affecte le secteur judiciaire, posent bien évidemment la question «de la place de la justice dans l'architecture des pouvoirs étatiques ${ }^{6}$, de sa singularité et de son rapport aux autres composantes de l'appareil étatique.

\section{Moderniser l'organisation judiciaire}

Le constat d'un « indéniable développement de la préoccupation gestionnaire au sein des juridictions $»^{7}$ semble aujourd'hui acquis : depuis les années 1980, par des mécanismes et des réformes diverses, la justice connaît un processus de prise en compte, de montée en puissance voire de valorisation du versant gestionnaire et administratif de son activité.

\section{a. Une politique en direction du justiciable devenu " usager"}

Elle a d'abord pris la forme d'une politique en direction de «l'usager », c'est-à-dire d'une politique destinée à permettre aux justiciables de mieux accéder à la justice, plus largement et dans de meilleures conditions. De nombreux rapports parlementaires ont en effet fait le constat d'une crise de la justice qu'il s'agissait d'enrayer, en toilettant dans un premier temps les apparences que la justice offrait à ceux qui s'adressaient ou souhaitaient s'adresser à elle. La simplification des procédures, la création de l'aide juridictionnelle entreprises à l'échelle de l'administration centrale étaient alors complémentaires de la mise en œuvre dans les juridictions d'une politique d'accueil des justiciables. Cette politique de modernisation s'appuyait sur une conception relativement restreinte et même restrictive de la modernisation, utilisant les outils classiques de l'Etat-Providence pour simplifier et démocratiser l'accès aux tribunaux et plus largement au droit, réduire la distance culturelle entre les professionnels et les profanes qui se tournent vers eux, adapter à la marge les outils techniques et les méthodes de travail pour rendre le traitement des procédures plus rapide.

Ce n'est qu'à partir du milieu des années 1980 que s'opère un renversement de perspective. Alors que jusque-là, des causes principalement exogènes du malaise judiciaire étaient invoquées, à partir de 1988 et du « rapport sur l'état de la Justice » que l'Inspection des services judiciaires remet au Garde des Sceaux, l'appareil judiciaire est lui-même mis en cause et directement rendu responsable des difficultés qu'il rencontre. Ce constat appelle dès lors des réformes plus profondes touchant cette fois aux structurations fondamentales sur lesquelles reposent l'institution et la culture judiciaires. Mais c'est toujours au nom de «l'usager », de la nécessité d'assurer une qualité du service public due au justiciable que la nécessité d'un changement organisationnel en profondeur va être justifiée. Alors que la Justice a une forte tradition d'indépendance et de revendication de sa singularité, les modernisateurs de la période 1988-1993 développent une distinction sémantique entre « institution judiciaire » et « administration judiciaire » ou encore entre « rendre la justice » et

\footnotetext{
${ }^{6}$ CADIET L. (2003), « Introduction », in CADIET L. et RICHER L. (dir.), Réforme de l'Etat, réforme de la justice, Puf, Paris.

CHRISTOPHE TCHAKALOFF M.-F., DESCHAMPS T. et MOUHANNA C. (2001), La spécificité de l'administration française de la justice: première approche, rapport de recherche de l'Institut international d'administration publique pour la Mission de recherche Droit et Justice, Paris, p.66.
} 
« administrer les moyens de la justice », ce qui leur permet alors de légitimer le discours de modernisation du service public de la justice et d'envisager la réforme du fonctionnement de la machine judiciaire mais aussi la remise en cause de ses spécificités les plus marquées et symboliques - telles que la carte judiciaire ${ }^{8}$.

\section{b. Des réformes visant à " mieux administrer " la justice}

A partir de là, toute une série de réformes vont être entreprises avec plus ou moins de succès. La structure du ministère de la Justice elle-même va connaître des transformations : renforcement des directions ou acteurs horizontaux (transformation du Service de l'administration générale en Direction des affaires générales et de l'équipement, consolidation de l'Inspection des services judiciaires...) et création d'instances de modernisation (Modac puis Mission modernisation) chargés de mettre en place une cohérence transverse aux différentes directions verticales et cloisonnées que sont la Direction des services judiciaires, la Direction des affaires criminelles et des grâces et la Direction des affaires civiles et du sceau ${ }^{9}$, et de diffuser une culture de gestion, dans un univers de magistrats traditionnellement plus sensibles au droit et à la dimension juridique de leurs missions - en juridiction comme lorsqu'ils sont détachés en administration centrale - qu'à une rationalité de type gestionnaire.

Localement, il est procédé à la fin des années 1990 à la création des Services administratifs locaux (SAR), pour déconcentrer à l'échelon régional de la Cour d'appel la gestion des moyens humains et matériels de l'ensemble des juridictions du ressort. Les SAR sont maintenant les principaux espaces où peut s'exprimer et se mettre en place une logique administrative de rationalisation des moyens, de même que la Cour d'appel semble être l'échelle territoriale la plus en mesure de porter une logique de recherche "d'efficacité" et "d'efficience" de l'activité judiciaire ${ }^{10}$. Plus récemment encore, la mise en place d'un système de primes au mérite pour les magistrats du siège, géré par le premier président de la Cour d'appel, contribue à renforcer ce niveau territorial et à donner aux chefs de cours des outils d'évaluation et de contrôle de l'activité des juges ${ }^{11}$.

Parallèlement, l'adoption de nouvelles règles de comptabilité publique centrées autour de l'évaluation de la performance par rapport à des objectifs (avec l'application de la Loi d'orientation des lois de finances et sa mise en œuvre actuellement) ${ }^{12}$ constitue une des manifestations les plus visibles de cette logique d'économie de moyens mais aussi de meilleure utilisation des moyens disponibles, mêlant recherche d'efficacité et recherche d'une plus grande accountability de l'activité judiciaire envers les acteurs politiques et parlementaires. La réflexion sur la qualité de la justice d'une part et celle sur la mesure de la performance des juridictions d'autre part sont les deux versants d'une même question, celle de l'efficacité d'un appareil de justice qui fonctionne à la fois sur le plan de la mise en œuvre d'une valeur (le juste) et sur celui de l'administration d'un pouvoir ou d'une autorité par un

\footnotetext{
${ }^{8}$ COMMAILLE J. (2000), Territoires de justice. Une sociologie politique de la carte judiciaire, Puf, Paris.

${ }^{9}$ Auxquelles il faut ajouter la Direction de l'administration pénitentiaire et la Direction de la protection judiciaire de la jeunesse mais dont il n'est pas question ici, puisque notre propos se limite à ce qui a trait aux instances judiciaires à proprement parler.

${ }_{10}$ Sur cette distinction entre efficacité et efficience dans le secteur judiciaire et sur la façon dont elles conditionnent la légitimité de la justice, voir VIGOUR C. (2004), «Légitimité, efficacité et réformes de la justice », communication au colloque « Justice pénale et nouvelles technologies », Grenoble, 17 décembre 2004.

${ }^{11}$ Sur un plan présenté comme strictement administratif et non juridictionnel. Ceci dit, rien ne dit que ce type d'outil ne peut pas être utilisé comme un moyen de récompenser des allégeances et / ou de pénaliser certaines pratiques qui ont trait à des modes de gestion de dossier (surtout à l'instruction). C'est en tout cas l'argument développé par un certain nombre d'acteurs individuels ou collectifs hostiles à cette mesure, dont le Syndicat de la Magistrature, au nom de la spécificité des missions du juge du siège dont l'indépendance doit être en tous points garantie.

${ }^{12}$ Voir le numéro spécial de la Revue française de Finances publiques, $\mathrm{n}^{\circ} 88,4,2004$ consacré à cette question et en particulier la contribution de Didier Marshall sur l'application de la LOLF au niveau des TGI.
} 
dispositif de type bureaucratique. Elles témoignent des tensions entre une approche par des indicateurs quantitatifs relatifs à une gestion de l'activité judiciaire considérée comme un process de type bureaucratique et une approche mettant l'accent sur l'acte juridictionnel, sur la façon de rendre justice ${ }^{13}$.

La promotion par quelques modernisateurs d'une logique gestionnaire, administrative et managériale à l'échelle de la Justice a donc percuté de fait les références et modes de fonctionnement classiquement institués. L'action judiciaire, traditionnellement appréhendée en termes de problèmes ou d'énigmes juridiques qu'il faut régler, dénouer, démêler (trouver la bonne décision, bien argumentée, bien charpentée qui respecte à la fois des exigences de justice et de légalité) s'est doublée d'une problématique relative à la bonne administration de la justice, à la maîtrise et à l'utilisation des ressources, à l'évaluation de l'activité mesurée à l'aune de l'écoulement des stocks de dossiers, de la gestion des flux et de la satisfaction des usagers... de la même façon que la problématique de l'acte juridictionnel et donc de l'indépendance qu'il suppose a été percutée par une logique de nature gestionnaire et administrative, traditionnellement distincte et considérée comme secondaire, aux antipodes de ce qui forme l'éthos classique du magistrat, et ce, d'autant que cette rationalisation du fonctionnement de l'institution s'est également effectuée par le biais de la diversification de la gamme des services et des procédures judiciaires ou para-judiciaires disponibles.

\section{Inventer ou réinventer de nouvelles formes de justice}

Ces formes de justice et procédures ont en commun d'apporter une réponse judiciaire théoriquement plus systématique parce que plus diversifiée; d'accélérer les temporalités judiciaires et en un mot de gagner du temps, quitte à repenser les formes les plus canoniques de l'exercice de la justice, telles que l'audience publique

\section{a. La diversification des acteurs et lieux de justice}

Repliée sur elle-même, réclamant un statut spécifique à la hauteur des tâches qui sont les siennes, l'institution judiciaire s'inscrit traditionnellement dans une logique d'autonomie et d'exceptionnalité. Indépendante mais aussi distante, théâtrale et sacralisée, elle se donne à voir comme un milieu fermé qui fonctionne autour de professionnels spécialisés maîtrisant son langage, ses rituels, et une même compétence juridique. Le Palais de justice, lieu emblématique de l'institution, symbolise bien cette " tour d'ivoire » dans laquelle évoluent les praticiens mais que connaissent peu les citoyens "ordinaires".

Pourtant, aujourd'hui et depuis le début des années 1990, en matière civile comme en matière pénale, on note la diversification des formes, lieux et acteurs de justice qui participent du processus judiciaire : la médiation, la conciliation et les procédures dites de troisième voie se développent, conduisant à laisser une place accrue à des acteurs nouveaux ou redécouverts (les délégués du procureur, les médiateurs ou les conciliateurs de justice ${ }^{14}$ ). Les procédures telles que le référé, la citation directe, le traitement en temps réel, la comparution immédiate ${ }^{15}$ ou plus récemment encore la comparution préalable sur reconnaissance de culpabilité également appelée plaider-coupable - indiquent que l'urgence est devenu «principe et non

\footnotetext{
${ }^{13}$ La Mission de recherche Droit et justice a lancé un appel d'offre « Qualité de la justice » qui témoigne bien de l'actualité de la question de l'évaluation du service rendu par les tribunaux. Voir notamment CAVROIS M.-L., DALLE H. et JEAN J.-P. (dir.) (2002), La qualité de la justice, La Documentation française, Paris.

${ }^{14}$ MILBURN P. (2002), La médiation: expériences et compétences, la Découverte, Paris ; CHEVALIER P., DESDEVISES Y., MILBURN P. (dir.) (2003), Les modes alternatifs de règlement des litiges: les voies nouvelles d'une autre justice, la Documentation française, Paris.

${ }^{15}$ BRUNET B. (1998), « Le traitement en temps réel : la Justice confrontée à l'urgence comme moyen habituel de résolution de la crise sociale », Droit et société, 38, p.91-107.
} 
plus exception $»^{16}$ au sens où « la procédure d'urgence tend à devenir, pour partie au moins, une voie de délestage, une procédure vers laquelle s'orientent naturellement ce qui est urgent, mais aussi ce qui ne présente aucune difficulté particulière, qui ne soulève pas de contestation sérieuse $\gg{ }^{17}$.

Cette création de formes et de procédures permet d'accélérer le temps de réponse en allégeant le formalisme, en mettant en place des circuits courts entre police, parquet et tribunal; mais aussi de systématiser la réponse judiciaire, surtout en matière pénale où sont proposées des solutions semi-pénales comme la composition et la réparation pénales, le rappel à la loi, le classement sous conditions, qui sont autant d'intermédiaires entre le classement sans suite et la poursuite des infractions et qui reposent à la fois sur les substituts du procureur, ses délégués et sur des acteurs extérieurs, comme les médiateurs.

La politique immobilière et architecturale du ministère de la Justice est elle-même emblématique de cette transformation de la justice et de la multiplication des lieux de justice. Si depuis le début des années 1990 une politique de construction de nouveaux Palais a été entreprise et a signifié un retour à une certaine monumentalité judiciaire ${ }^{18}$, les significations et déclinaisons de cette monumentalité se sont diversifiées. La façon dont la salle des pas perdus de ces nouveaux Palais est pensée constitue à cet égard un excellent exemple. Le sens de ce lieu change: d'immense espace vide, peuplé seulement des professionnels de justice et symbolisant le passage entre l'arrivée dans le palais et l'entrée dans les salles d'audience, il devient un lieu d'accueil du public, conçu de telle façon que les justiciables se repèrent, $\mathrm{s}^{\prime}$ informent et que le cas échéant, ils puissent assister aux procès à grande échelle ${ }^{19}$. De même, l'implantation de maisons de la justice et du droit dans les quartiers dits difficiles et la création d'antennes de justice en périphérie ${ }^{20}$, témoignent d'une forme de diffusion par l'essaimage de lieux de justice et le développement d'une justice dite de proximitée ${ }^{21}$.

Parallèlement, les rapports que la justice entretient avec les autres espaces sociaux et politiques ont eux-mêmes évolué, bon an mal an, via la participation aux groupes locaux de prévention de la délinquance et la formulation, même limitée, d'une politique judiciaire de la ville $^{22}$. Ces évolutions sont en grande partie liées à ce double impératif auquel la justice doit faire face : gérer un contentieux qui a explosé depuis les années 1980 (et donc s'adapter pour évacuer davantage de dossiers, quitte à les déléguer à des acteurs parajudiciaires) et donner à voir aux acteurs politiques et à l'opinion publique que la justice contribue, manifestement, visiblement à la lutte pour la sécurité qu'elle doit mener désormais aux côtés d'autres acteurs publics ou privés.

\section{b. La diffusion des formes juridiques et judiciaires}

Un certain nombre de signes indiquent donc que la justice évolue dans son rapport à l'extérieur mais aussi en interne, dans les formes mêmes que prennent les juridictions et les procédures juridiques. Mais ce qu'il est intéressant de souligner ici, c'est le processus au fil

${ }^{16}$ DAVENAS L. (2003), «Le traitement de l'urgence : exception ou principe ? En procédure pénale », in CADIET et RICHER L. (dir.), (2003), op. cit., p. 174.

${ }^{17}$ NORMAND J. (2003), « Le traitement de l'urgence : exception ou principe ? Introduction », in ibid. p.168.

${ }_{18}$ JACOB R. (1994), Images de la justice. Essai sur l'iconographie judiciaire du Moyen Age à l'âge classique, Le Léopard d'or, Paris.

${ }^{19}$ GOUTTES B. (2002), « La nouvelle architecture judiciaire et l'évolution de la conception des palais de justice ", in La nouvelle architecture judiciaire. Des palais de justice modernes pour une nouvelle image de la justice, La Documentation française, Paris, p. 10.

${ }^{20}$ WYVEKENS A. (1996), « Justice de proximité et proximité de la justice. Les maisons de justice et du droit », Droit et société, 33, p. 363-388.

${ }^{21}$ WYVEKENS A. et FAGET J. (dir.) (2001), La justice de proximité en Europe : pratiques et enjeux, Erès, Ramonville Saint-Agne.

${ }^{22}$ DONZELOT J. et WYVEKENS A. (1998), La politique judiciaire de la ville : de la "prévention" au " traitement », Rapport de recherche pour la mission « Droit et Justice » / IHESI / DIV. 
duquel un certain nombre d'innovations se diffusent et s'implantent au sein du système français. La référence au New public management, la montée en force de cette logique et des préoccupations concrètes qui en découlent, nous semblent être un des ressorts qui permet de comprendre la diffusion de solutions et de formes juridiques que l'on doit alors considérer plus largement comme des solutions d'action publique qui circulent à l'échelle nationale et internationale.

Bien entendu, il ne s'agit pas de prétendre ici que les transformations actuelles de la justice seraient réductibles à la diffusion d'une logique managériale : bien d'autres arguments et modèles de bonnes justice s'affrontent au sein même des arènes sociales, politiques et judiciaires qui participent à la mise en œuvre de réformes de justice. Antoine Vauchez et Laurent Willemez ont bien montré, dans le cas de la réforme de la Cour d'Assises et des Tribunaux de commerce, le rôle important du modèle de procès défendu par la Convention européenne des droits de l'Homme dans le processus de constitution de communautés d'acteurs susceptibles d'appuyer et de rendre possible de telles réformes. L'article 6 de la Convention européenne des Droits de l'Homme et la notion de «procès équitable » sont mobilisés comme des ressources pour la formation de consensus réformateurs et la déspécification de mondes judiciaires cloisonnés.

Cela étant, ce que nous voudrions souligner ici, c'est la force de la logique gestionnaire qui " s'accroche " à des dispositifs juridiques, à des innovations technologiques et qui s'imprime en eux, les pénètre au point que la diffusion de ces innovations devient indissociablement diffusion de la logique managériale elle-même. La technologie est un bon exemple de ce processus de diffusion dans la mesure où les « réformateurs » actuels ont bien compris que l'informatique et les technologies d'information et de communication pouvaient être appréhendées comme des leviers privilégiés d'évolution des pratiques et des modes de fonctionnement internes à l'institution. Le cas de la visioconférence - objet d'une recherche en cours $^{23}$ - peut illustrer notre propos. Il ressort en effet d'une analyse de la genèse de la visioconférence utilisée pour pratiquer des actes judiciaires (tenue d'audiences civiles et pénales, interrogatoires de première comparution, auditions de témoins...) que son utilisation a été motivée initialement par la nécessité de débloquer une situation spécifique particulièrement complexe - en l'occurrence à Saint-Pierre-et-Miquelon - et ensuite principalement justifiée par des arguments de type gestionnaire (économiser les ressources publiques, ne pas multiplier des déplacements dont la nécessité, dès lors qu'elle était mise en regard du coût, pouvait perdre de son absolu et être relativisée $)^{24}$

Ce qu'il est intéressant de noter c'est le mécanisme à partir duquel la technologie devient porteuse de la logique gestionnaire, qu'elle véhicule ensuite constamment. Parce qu'il est plastique, le support technologique se charge d'un sens managérial, d'une interprétation en termes d'économies de moyens qu'il ne comporte pas forcément intrinsèquement, mais que le déploiement d'argumentaires de justification va rendre possible, crédible même et attacher durablement à lui ${ }^{25}$. Précisons en effet que si le discours des acteurs est invariablement centré autour de l'argument d'une économie de moyens permise par l'utilisation de la

\footnotetext{
${ }^{23}$ Il s'agit d'une recherche collective intitulée « Justice et TIC : la gestation d'un nouveau modèle de justice ? », effectuée dans le cadre de l'Action concertée incitative (ACI) «Terrains, techniques, théories: Travail interdisciplinaire en sciences sociales » du ministère de la Recherche et financée par le Fonds national de la science.

${ }^{24}$ «La visioconférence permet des économies de moyens à la fois humains (déplacement des magistrats parisiens) et financiers (billets d'avions...) : c'est le cas à Saint-Pierre-et-Miquelon [...]. La visioconférence correspond d'abord à une visée d'économie et ensuite se greffe une perspective plus qualitative. ", extrait d'entretien avec un magistrat, Cour d'appel de Paris, 19 juillet 2004.

${ }^{25}$ DUMOULIN L. et LICOPPE C. (2004), « Juger à distance : des conditions d'émergence de la visioconférence dans la justice française », communication au colloque « Justice pénale et nouvelles technologies », CERDAP / CERAT / ENM, Grenoble, 17 décembre 2004.
} 
visioconférence, cela n'est en rien démontré. A aucun moment d'ailleurs, personne n'a tenté d'évaluer la réalité des gains escomptés par le recours à un dispositif de visioconférence comparé aux déplacements physiques. La logique managériale "colle désormais à la peau" de cet outil, au point que l'élargissement de la possibilité de recourir à la visioconférence - dans des cas aussi différents que le contentieux des étrangers en situation irrégulière ou que le contentieux de la détention - est complètement indexé à de possibles économies de moyens, raccordé à des problématiques - comme celle de l'escorte des détenus convoqués par le tribunal - qui sont repensées essentiellement sous l'angle de la rationalisation des pratiques et de la maîtrise des dépenses. Le point important, s'agissant d'ailleurs de réformes aussi différentes que l'introduction du plaider-coupable dans la procédure pénale française ou que l'utilisation de la visioconférence, porte sur la transformation de formes classiques telles que le procès en co-présence ou le principe de l'audience publique : qu'il s'agisse de juger à distance ou bien de conclure un accord avec le représentant du ministère public qui sera ensuite homologué par le juge du siège, ce qui est questionné et possiblement redessiné, c'est le rôle du tribunal, du procès et de l'audience. La forme ne peut être dissociée du fond : ce qui peut sembler comme le plus technique ou le plus administratif n'est jamais exempt d'effets sur la façon dont est rendue la justice et partant, sur son rôle comme arbitre social et politique.

C'est donc ainsi, à travers des réformes et innovations disparates, tantôt apparemment insignifiantes et limitées, tantôt ouvertement ambitieuses voire radicales, que s'effectue concrètement la diffusion d'une logique managériale, d'un référentiel modernisateur ou encore d'un "sens commun réformateur ${ }^{26}$ portés par des acteurs appartenant à différentes sphères judiciaires, sociales et politiques qui ont en commun de défendre une certaine vision de la justice. A l'opposé, un certain nombre d'acteurs, individuels ou collectifs, mettent l'accent sur la spécificité des missions judiciaires pour rejeter la logique gestionnaire qui gagne l'espace de justice comme elle s'impose dans d'autres secteurs de l'action publique. La justice ne saurait être soumise à la même logique comptable, soupçonnée d'être uniforme ou uniformisante, dans la mesure où ce que les acteurs judiciaires produisent n'est ni standardisé ni standardisable, et ne saurait être réduit à un processus bureaucratique mesurable ${ }^{27}$. Le positionnement de l'expertise dans la justice, incarne, d'une certaine façon, cette tension entre logique de l'efficacité (conformité à des objectifs) et logique politique (choix par valeur), et en fait donc un prisme intéressant pour observer les dualités et difficultés concrètes que rencontre une logique de l'efficacité qui s'installe progressivement dans le milieu judiciaire.

\section{II / L'expertise entre logique juridique et logique de l'efficacité}

En effet, la conception juridique de l'expertise a été progressivement élaborée autour d'une définition permettant de préserver les fondements politiques d'une maîtrise du jugement et du procès par le juge, professionnel patenté que sa maîtrise du droit rend légitime à rendre justice. Pourtant, une approche sociologique du recours à l'expertise montre que celle-ci fonctionne de fait comme un des instruments et facteurs d'adaptation de l'institution à une exigence accrue d'efficacité et de rationalisation des modes d'organisation, et ce bien qu'elle soit intrinsèquement vecteur de complexification du procès, de ralentissement des délais et d'augmentation des coûts - et traditionnellement perçue comme telle. Les tiraillements entre

\footnotetext{
${ }^{26}$ VAUCHEZ A., WILLEMEZ L. [et al.], (2004), Les " mondes judiciaires » et la construction d'un horizon réformateur commun, Rapport de recherche Mission de recherche Droit et justice / Curapp.

${ }^{27}$ Voir par exemple les positions du Syndicat de la Magistrature telles qu'elles sont exprimées par son secrétaire général, dans SAINATI G. et SCHALCHLI U. (2001), «Le taylorisme à l'assaut de la justice », Justices, 167, avril.
} 
ces différentes logiques nous semblent emblématiques des tensions qui travaillent actuellement l'espace judiciaire.

\section{De l'élaboration d'une identité « technicisée » de l'expert}

Si le recours à l'expertise judiciaire s'est intensifié et banalisé pendant les XIXe et XXe siècles, le discours sur ce que doit être l'expertise, sur la place qu'elle doit occuper dans le processus judiciaire n'a pas changé : c'est une même vision politique de la justice et de l'expertise qui s'exprime au fil d'un cadre normatif constamment enrichi.

\section{a. Une définition décisionniste de l'expertise judiciaire}

L'histoire de l'expertise judiciaire est l'histoire d'une perpétuelle tentative d'encadrement et de normalisation du recours à des savoirs et acteurs non juridiques dans le procès. Au fur et à mesure que s'accentuent la massification des litiges soumis à la justice d'une part et leur complexification d'autre part, l'institution a été amenée à préciser les attributions prioritaires des différents acteurs prenant part à l'action judiciaire, et en particulier celles du magistrat, acteur central du dispositif. On peut dire que l'intégration verticale des différentes opérations constitutives du règlement d'un conflit a été délaissée au profit d'une spécialisation des différents intervenants : au juge la tâche de dire le droit, au greffier celle d'administrer les contentieux, à l'expert celle de résoudre les questions techniques, sous la stricte responsabilité du juge.

En l'occurrence, ce que nous révèle l'étude diachronique de l'expertise, c'est la permanence et le renforcement d'un modèle normatif centré autour d'un découpage strict entre ce qui relève de la connaissance technique et ce qui relève de la décision; entre l'expert, technicien prestataire de service, et le juge, complètement maître du processus judiciaire et de son déroulement. Les rôles respectifs sont clairement dessinés et distingués : en matière civile, le juge décide de l'opportunité de réaliser une expertise, il désigne l'expert c'est-à-dire qu'il le choisit nommément mais que, ce faisant, il choisit aussi de quelle discipline et de quelle spécialité il doit relever (chirurgien dentiste ou stomatologue? Ingénieur ou architecte?). C'est encore lui qui indique la nature des investigations à mener, le libellé de la mission qu'il peut ensuite librement accroître ou restreindre. Il dispose en outre d'un pouvoir de contrôle sur l'expert pendant les opérations et la phase d'expertise à proprement parler. In fine, lorsque le rapport est rendu et la mission terminée, le juge a toute liberté pour suivre ou non le diagnostic, l'avis ou les recommandations de l'expert : c'est à lui qu'il revient d'apprécier les résultats de l'expertise.

C'est ainsi que le modèle juridique de l'expertise judiciaire repose sur une véritable dissociation du juge et de l'expert, le premier disposant du pouvoir d'impulsion, de contrôle et d'évaluation; le second effectuant des fonctions techniques d'exécution d'une mission. Comme le résume Michel Olivier, ancien magistrat, il s'agit pour l'expert d'accomplir « la mission, rien que la mission mais toute la mission $»^{28}$. Cette conception décisionniste de l'expertise ${ }^{29}$ repose en fait sur l'idée selon laquelle le juge doit superviser l'ensemble du processus judiciaire: les acteurs, qu'il sollicite, les experts en particulier, mais aussi les médiateurs, ne peuvent intervenir qu'à partir du moment où ils réfèrent de leur action au juge professionnel. C'est bien là le cadre à la fois légal et légitime de leur action.

\section{b. Des experts "exclus" de l'organisation judiciaire}

Cette idée d'un rôle limité et borné de l'expert va de pair avec une certaine idée du statut de l'expert, de sa place dans l'institution judiciaire elle-même. L'histoire de l'expertise

\footnotetext{
${ }^{28}$ OLIVIER M. (1990), De l'expertise civile et des experts, Tome 1, Berger-Levrault, Paris, et (1995), Tome 2 , Berger-Levrault, Paris.

${ }^{29}$ HABERMAS J. (1973), La technique et la science comme idéologie, Gallimard, Paris.
} 
est aussi l'histoire d'une critique de ses dysfonctionnements, les experts étant constamment soupçonnés de provoquer l'augmentation des coûts du procès, l'allongement des délais et la multiplication des niches procédurales contribuant à accroître l'insécurité juridique.

C'est ainsi que la Chancellerie s'est toujours fermement opposée à la création d'un corps d'experts professionnalisés et a régulièrement appelé les magistrats à une grande modération dans leurs recours aux experts. En principe, l'expertise judiciaire reste une activité occasionnelle. Les experts peuvent être inscrits sur des listes mais leur inscription ne leur confère pas de monopole sur l'activité d'expertise. Ils sont seulement porteurs d'un titre protégé - «Expert près la cour d'appel de ... » ou «Expert agréé par la Cour de cassation » mais auquel peu de prérogatives spécifiques sont attachées. En effet, leur inscription n'implique pas qu'ils soient effectivement missionnés pour telle ou telle affaire précise. De facto, les listes regorgent de noms de personnes qui, en pratique, ne font jamais ou presque de missions d'expertise judiciaire.

La réalité des besoins en savoirs spécialisés a donc amené l'institution à s'entourer d'experts et à les solliciter de plus en plus. Toutefois celle-ci s'est durablement et constamment efforcée, via l'adoption d'un dispositif juridique draconien, de les maintenir à distance et sous contrôle, octroyant la maîtrise exclusive du processus décisionnel aux magistrats professionnels et réaffirmant régulièrement leur nécessaire suprématie ${ }^{30}$. Ce qui nous semble le plus notable dans l'élaboration de cette définition juridique de l'expertise judiciaire - telle qu'elle s'est progressivement sédimentée -, c'est que cette vision de l'expert comme prestataire de service ponctuellement au service de la justice, et de l'expertise comme procédure neutre et indolore parce qu'encadrée par le droit et le juge, est parvenue à fonder toute la culture juridique de l'expertise. Élaborée par une succession de textes législatifs et réglementaires, incorporée par la doctrine, actée et mise en œuvre par la jurisprudence, cette conception a progressivement pris forme pour devenir une réalité normative contraignante, un socle évident, un "déjà-là" et un "allant de soi" des représentations spécialisées et profanes de l'expertise. Il structure, aujourd'hui encore, très fortement les représentations de l'expertise en justice, celle-ci étant véritablement perçue comme un facteur perturbant, une sorte d'appendice, de mal nécessaire au sein du processus judiciaire

\section{Des usages et paradoxes de l'expertise en situation}

Pour autant, une analyse des pratiques des magistrats donne à voir des usages concrets de l'expertise qui s'émancipent des dispositions juridiques. L'expertise permet au magistrat de gagner du temps (au sens d'économiser son temps), de s'épargner certaines tâches et conséquemment de se concentrer sur ce qu'il estime devoir être son activité principale, en l'occurrence dire le droit et rendre la justice. En déléguant certaines missions à l'expert, en lui conférant une fonction de contact avec le terrain ou de médiation avec les parties, le magistrat se ménage de petites marges de manœuvre dans la gestion quotidienne de sa charge de travail. Mais le développement de l'expertise continue de poser problème puisqu'il entre en contradiction avec une rationalité comptable qui considère l'expertise comme un facteur d'allongement des délais, de multiplication des procédures et d'augmentation des coûts.

\section{a. L'expert, un contact privilégié avec le terrain}

Convoqué en tant que spécialiste d'une matière précise, le technicien observe et interprète une situation qu'il a pour mission de décoder à partir de ses propres compétences techniques, c'est le sens et l'utilisation première qui est faite de l'expertise. Ceci dit, le

${ }^{30}$ CHAUVAUD F., avec la collab. de DUMOULIN L. (2003), Experts et expertises judiciaires en France, XIXe et XXe siècles, PUR, Rennes, chapitres 1 à 3 . 
rapport offre concomitamment au tribunal un exposé de la cause, de ses tenants et de ses aboutissants, ainsi qu'une présentation des protagonistes et leurs revendications. L'encombrement judiciaire, la tendance des juges à se concentrer sur les aspects juridiques de leur travail peuvent expliquer qu'ils aient assez peu le temps et / ou l'envie de se déplacer sur le terrain pour voir d'eux-mêmes comment les choses se présentent et qu'ils se satisfassent de fait des données recueillies par l'intermédiaire de l'expert qu'ils ont désigné. En matière familiale comme dans les litiges automobiles ou encore le contentieux des loyers commerciaux, il n'est pas rare que le juge délègue ouvertement ses fonctions d'audience des parties en confiant à l'expert le soin de prendre connaissance pour lui, de la dimension concrète du litige, d'observer les lieux, d'entendre les parties et leurs visions respectives du litige. L'expertise apparaît ici investie comme une occasion de disposer d'un état des lieux, au sens propre comme au sens figuré, de la situation conflictuelle. L'expert est placé d'emblée dans une sphère non seulement technique mais bien plus largement factuelle. La délégation de la dimension concrète du dossier permet au magistrat de se concentrer sur les aspects procéduraux et juridiques, à partir d'une appréhension essentiellement écrite de la réalité. A bien des égards donc, l'expert remplace le magistrat dans les missions de contact avec les parties et assume une grande partie du travail de terrain.

\section{b. L'expert, un médiateur tout trouvé}

Ce processus de délégation d'un certain face-à-face avec les parties se double d'un certain transfert à l'expert d'une activité de gestion du conflit, c'est-à-dire en l'occurrence de la médiation des parties. Les missions explicites de médiation s'adressent le plus souvent aux psychologues dans le cadre d'affaires de la famille, mais l'usage de l'expert comme d'un médiateur concerne aussi d'autres types de savoirs et de secteurs professionnels (en particulier ceux de la construction). Parce qu'il est investi d'une mission de justice, qu'il est installé dans une position d'extériorité par rapport au conflit, l'expert peut intervenir pour limiter les demandes des uns ou des autres, calmer le jeu, proposer des solutions concrètes. Bien que le Code de procédure civile défende explicitement au juge de lui donner mission de concilier les parties, l'expert peut, de fait, œuvrer pour une conciliation, que ce soit de sa propre initiative ou bien suite à une sollicitation informelle du juge. Le juge est en effet autorisé à constater et valider un protocole d'accord intervenu pendant la phase d'expertise. Concrètement, il arrive que certains juges programment une descente sur les lieux litigieux, accompagnés d'un expert, et ce avec la volonté ouverte de tenter une opération de règlement amiable du désaccord. Il s'agit alors d'inciter voire de persuader les parties de l'intérêt de trouver un terrain d'entente. L'expert contribue donc à faire sortir un certain nombre d'affaires du circuit judiciaire, en leur apportant une solution négociée, validée ensuite par le juge.

Sur ce double plan, la procédure d'expertise produit donc des effets utiles pour l'institution judiciaire envisagée d'un point de vue organisationnel. Elle contribue à réguler le fonctionnement d'une machine confrontée à la massification du contentieux. Certes, le phénomène est probablement assez limité quantitativement - quoique les connaissances sur ce point soient assez défaillantes ${ }^{31}$ - mais, en tout cas, restreint ou plus conséquent, ce phénomène indique une tendance et met en lumière certains usages stratégiques de l'expertise. L'expertise apparaît donc comme un outil utile dans le cadre d'un fonctionnement rationalisé de l'institution judiciaire : elle participe indirectement à la prise en charge d'impératifs gestionnaires, rendus d'autant plus sensibles que la masse du

\footnotetext{
${ }^{31}$ En effet, les informations dont on dispose sont anciennes et très parcellaires. Voir l'enquête la plus récente : MINISTERE DE LA JUSTICE, LE TOQUEUX J.-L. (1993), Les expertises judiciaires civiles et administratives. Coûts et délais, enquête 1992, Ed. DAGE / Ministère de la Justice, Paris.
} 
contentieux s'accroît visiblement et qu'un cruel manque de moyens se fait $\operatorname{sentir}^{32}$. Mais ce n'est qu'en contournant un certain nombre de dispositions juridiques que ce type de pratique est possible et l'on voit dès lors que ces adaptations placent les magistrats (ainsi que les experts) en étau entre ce que le droit dit de la juste place de l'expert dans le processus judiciaire et ce qui apparaît comme une indispensable amélioration de l'organisation du travail judiciaire, en situation.

Toutefois, le plus intéressant est encore de remarquer que ce développement de l'expertise qui vise à répondre à des besoins de souplesse organisationnelle, percute de plein fouet la stricte logique économique, gestionnaire et comptable, puisqu'il contribue à une inflation des moyens et délais nécessaires, là où une réduction et une rationalisation des coûts sont recherchées. Les arrangements organisationnels qui contribuaient à donner de la souplesse au système et à permettre de gérer concrètement la pénurie de moyens sont remis en cause au nom d'une logique comptable qui risque dès lors de déstabiliser les équilibres en place, sans forcément parvenir à en proposer d'autres.

C'est ainsi que l'expertise donne à voir un paradoxe qui incarne bien les difficultés et ambiguités que comporte l'introduction d'impératifs managériaux dans la justice. Si la banalisation des savoirs savants dans le champ judiciaire contribue à alimenter une réorganisation, très limitée mais palpable à l'échelle des individus, des méthodes de travail et de la répartition des tâches au sein du processus judiciaire, il n'en reste pas moins que les attentes juridiques et politiques autour de l'expertise sont bien celles qui correspondent au modèle idéal de l'expertise judiciaire, c'est-à-dire à un encadrement et une maîtrise de l'expert par le juge que les impératifs managériaux ne font que renforcer mais que les magistrats remettent constamment en cause dans l'exercice de leur métier.

A l'heure de conclure, nous voudrions revenir sur les conflits qui peuvent survenir entre une logique de "l'efficacité" et une logique juridico-politique de construction de l'acceptabilité de l'action judiciaire. Nous avons vu que les magistrats s'ajustent, adaptent leurs comportements aux contraintes qui sont les leurs en situation (ne pas perdre de temps, déléguer pour gagner du temps et desserrer l'étau qui les étreint...). En ce sens, l'expertise est une des variables d'ajustement disponibles - parmi bien d'autres. Cela étant, les magistrats entrent alors en conflit avec les fondements d'une conception juridique et politique de l'expertise constamment réaffirmée par la Chancellerie. Travailler cet objet nous a permis de mettre en évidence le caractère contradictoire des injonctions auxquels ils sont soumis : améliorer leur productivité tout en maintenant intact le respect des procédures juridiques et en n'abdiquant pas ce qui relève de leur mission centrale, à savoir la pleine et entière maîtrise $d u$ procès. Bien entendu, cette double injonction s'avère intenable : ce n'est qu'en trouvant des raccourcis processuels, qu'en hiérarchisant les lieux et les moments où leur présence est exigée, en un mot en trouvant des « ficelles », qu'ils peuvent arriver à conjuguer cette double logique de la juridicité et de l'efficacité. Cette remarque est d'ailleurs valable tant pour les greffiers que pour les magistrats : si les seconds utilisent l'expertise pour s'épargner certaines tâches ou bien s'ils s'abstiennent de motiver leurs jugements correctionnels dès lors qu'ils ont la certitude qu'il n'y aura pas appel, les premiers n'assistent pas à toutes les audiences afin de vaquer à d'autres occupations et là encore, être plus "efficaces", gagner de ce temps si précieux. Négliger certaines missions estimées secondaires pour se concentrer sur celles qui apparaissent plus importantes, plus urgentes ou moins malléables, voilà ce qui permet effectivement de conjuguer des injonctions au moins partiellement contradictoires et qui se négocie notamment au fil des activités quotidiennes.

\footnotetext{
32 JEAN J.-P. (1997), « La judiciarisation des questions de société », Après-demain, octobre.
} 
